\title{
Noninvasive Visualization of Three-Dimensional Atrial Electrical Excitation Using Anterior and Posterior Magnetocardiogram
}

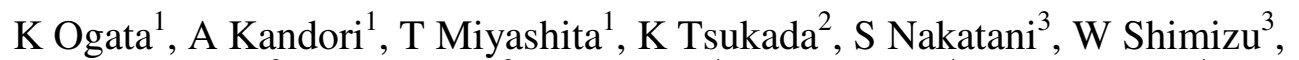

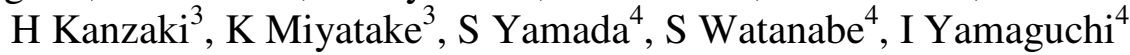 \\ ${ }^{1}$ Hitachi Ltd., Central Research Laboratory, Tokyo, Japan \\ ${ }^{2}$ Department of Electrical and Electronic Engineering, University of Okayama, Okayama, Japan \\ ${ }^{3}$ National Cardiovascular Center, Osaka, Japan \\ ${ }^{4}$ Institute of Clinical Medicine, University of Tsukuba, Ibaraki, Japan
}

\begin{abstract}
The aim of our work is to visualize three-dimensional (3-D) images of atrial excitation. We obtained these images by projecting the anterior and posterior currentarrow map (CAM), derived from a magnetocardiogram (MCG), onto a 3-D standard heart model. We generated the projected CAM (PCAM) of early and late atrial depolarization phases for fourteen healthy subjects. During early and late atrial excitation, the high-current areas of all subjects were in the right atrium of the anterior surface, and the left atrium of the posterior surface. The averaged ratio of the maximal current value of the early p-wave to the maximal current value of the late $p$-wave for all subjects was $0.9 \pm 0.2$. Furthermore, the averaged maximal current directions of the early and late $p$-wave were $78 \pm 15$ and $165 \pm 17$ degrees, respectively. Thus, we found that the PCAM could provide separated images of the left and right atrial excitations.
\end{abstract}

\section{Introduction}

Magnetocardiograms (MCGs) are a novel technology that can produce images of the cardiac electrical current. An $M C G$ measures the weak cardiac magnetic field generated by the adults [1] or by fetuses [2], noninvasively. A two-dimensional $(2-D)$ current-arrow map (CAM) [3] was developed as a practical method of calculating pseudo cardiac currents from an MCG. The CAM provides the foundation of a new diagnosis system for various heart diseases $[4,5]$. Furthermore, we were able to visualize multiple exited areas during ventricular excitation using anterior and posterior CAMs [6]. Recently, a novel method for projecting anterior and posterior CAMs onto a three-dimensional (3-D) standard heart model [7] was developed. This method is called $P C A M$ and gives us a clear view of the cardiac electrical current.

The aim of our work is to visualize 3-D images of atrial electrical excitation using PCAM. We used a superconducting quantum interference device (SQUID) system to measure the axial components of the anterior and posterior $M C G$ s. The anterior and posterior $C A M \mathrm{~s}$ were calculated by taking spatial derivatives of the measured anterior and posterior $M C G$ s. The 3-D standard heart model was generated from the magnetic resonance $(M R)$ images of three healthy subjects.

This standard heart model was then adjusted to the optimal position for each subject using information on the heart's position. This information took the form of the sinus node coordinates, which were calculated from $M C G$ signals. After adjusting the base current values of the anterior and posterior $C A M s$, they were projected onto the positionally corrected standard heart model. We applied $P C A M$ to obtain a cardiac-current image of the atrial depolarization phase (p-wave) of fourteen healthy subjects.

\section{Measurement}

\subsection{MCG measurements}

Figure 1 shows the conventional configuration for measuring $M C G$ signals. We used a Low-TC SQUID system (MC-6400, Hitachi High-Technologies Corporation), with 64 co-axial gradiometers to measure the $M C G$ signals, which were axial components to the chest wall (see figure 2). The magnetic flux resolution of all gradiometers was better than $20 \mathrm{fTHz}^{1 / 2}$. The SQUID sensors were laid out in an $8 \times 8$ matrix, with a pitch of 25 $\mathrm{mm}$, and the measurement area was $175 \times 175 \mathrm{~mm}$. For the anterior chest wall measurement, a sensor $(7,3)$ was placed above the position of the xiphoid process. For the posterior chest wall measurement, a sensor $(7,6)$ was placed directly underneath the position of the xiphoid process. The $M C G$ signals were acquired at a sampling 
frequency of $1 \mathrm{kHz}$ and passed through band-pass (0.1$100 \mathrm{~Hz}$ ) and power-line noise filters. The measurement period was $30 \mathrm{~s}$, and the $M C G$ signals for all pulses over this period were averaged. To adjust the phases of the anterior and posterior $M C G$ s, we simultaneously measured the Lead II electrocardiogram (ECG) signals.

\subsection{Heart MRI measurements}

To visualize the 3-D structure of the entire surface of the heart, $M R$ images were recorded by scanning $300 \mathrm{x}$ $300 \mathrm{~mm}$ coronal planes, vertically separated by $6 \mathrm{~mm}$ using an MRI system (Magnex 100 (1T), Shimadzu Corporation). We used ECG triggering (R-wave peak) to control $M R$ image capture. Body motions associated with the heartbeat were theoretically corrected by using $E C G$ triggering. The $M R$ examination for each subject was completed within about 30 minutes.

\section{Method}

We generated cardiac excitation images by projecting anterior and posterior $C A M \mathrm{~s}$, which are derived from anterior and posterior $M C G$, onto a 3-D standard heart model [7].

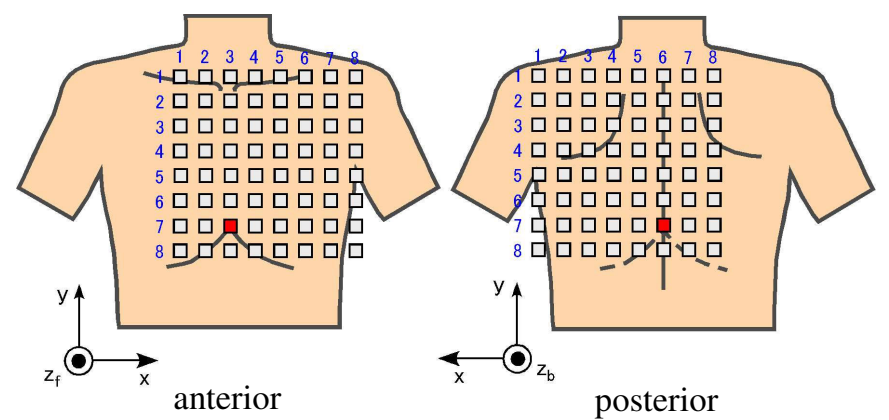

Figure 1. Configuration for MCG measurement.

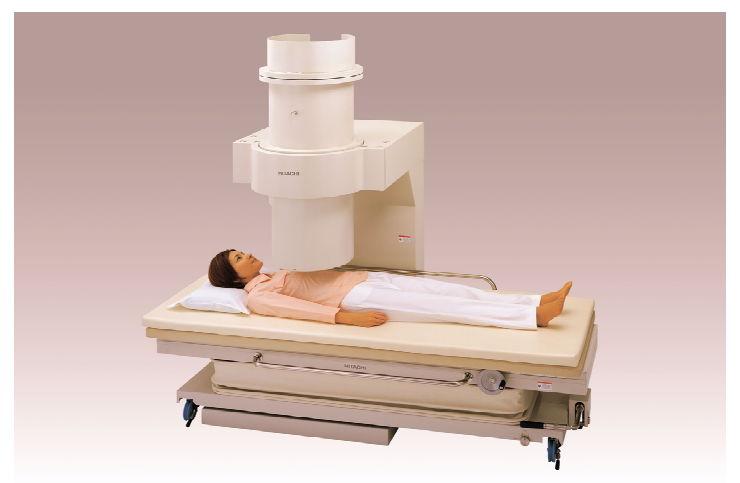

Figure 2. MCG system (MC-6400, Hitachi HighTechnologies Corporation).
We made the standard heart model from chest $M R$ images of the chest of three healthy subjects. After the $M R$ images were obtained, heart-outline points were extracted from each image and 3-D heart models for each subject were made from these points. The centroid of the heart model was calculated from all heart-outline points. Then, the distances from the centroid to each point were obtained. The averages were calculated from these distances for the three subjects and were used to generate the 3-D standard heart model.

The standard heart model was adjusted to the optimal position for each subject using information about the heart's position, in the form of the sinus node coordinates. The coordinates are obtained by applying an equivalent current dipole (ECD) algorithm [8] to the $M C G$ signals at the beginning of the p-wave. Therefore, the optimal position of the standard heart model was determined by superposing the position of the sinus node on the standard heart model from the calculated position of the corresponding current dipole.

The $C A M$ represents a $2-D$ pseudo cardiac electrical current [3]. The current arrows of $\operatorname{CAM}\left(\boldsymbol{I}=\left(I_{x}, I_{y}\right)\right)$ were calculated by taking orthogonal partial derivatives of the axial components to the chest wall of the cardiac magnetic field. In other words, the $C A M$ was obtained from $I_{x}=d B_{z, n} / d y$ and $I_{y}=-d B_{z, n} / d x$, here, $B_{z, n}$ represents the axial components of the measured cardiac magnetic field. Furthermore, the magnitude of the current arrow $|I|$ was derived from $|I|=\left(\mathrm{I}_{\mathrm{x}}^{2}+\mathrm{I}_{\mathrm{y}}^{2}\right)^{1 / 2}$.

The anterior and posterior $C A M s$ were projected onto the anterior and posterior surfaces of the positionally adjusted standard heart model, respectively. However, the projected CAM (PCAM) showed a discontinuous current pattern at the junction of the anterior and posterior surfaces of the standard heart model, because the base currents of the anterior and posterior $C A M s$ were quite different. This is because the distance between the heart and anterior measurement plane is not equal to the distance between the heart and posterior measurement plane. Therefore, we used a weighting coefficient $W$, which adjusted the base currents of the anterior and posterior CAMs. The weighting function was obtained by equalizing the anterior and posterior $C A M$ on the outermost heart outline for the xy plane.

To evaluate the PCAM of the atrial depolarization phase (p-wave), we extracted the maximal current value and direction. The maximal current value reflects the amplitude of electrical current in the myocardium. The maximal current direction reflects the electrical axis of the heart Therefore, PCAM could be verified by investigating these values in healthy subjects. The maximal current directions were quantified based on the definition of the current arrow direction (see Figure 3). 


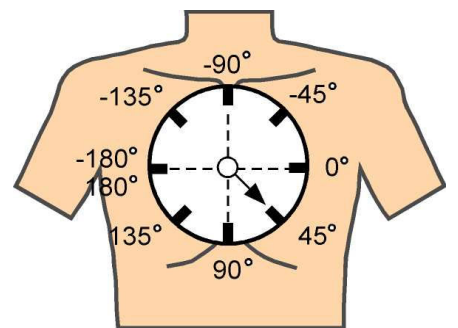

Figure 3. Definition of current arrow directions.

\section{Results}

\subsection{PCAM for typical healthy subjects}

Figure 4 shows the $M C G$ waveforms of the anterior and posterior measurements for one healthy subject. Figures 4 (a) and (b) show the posterior and anterior overlapped waveforms of all 64 channels. We can see that the $M C G$ waveforms were composed of the p-wave, QRS-complex, and T-wave. The lines in Figs. 4 (a) and (b) represent the two phases (\#1: early p-wave, \#2: late pwave) when the two PCAM images in figures 5 (a) and (b) were taken. Figures 5 (a) and (b) show PCAMs during the p-wave. The arrows indicate the current direction, and the color-map indicates the magnitude of the current vector.

The high-current area of the PCAM during the early pwave stafe (right atrial excitation, $80 \mathrm{~ms}$ ) was in the right atrium of the anterior surface of the standard heart model, and the current flow was in the inferior direction in the upper right. During the late p-wave (left atrial excitation, $110 \mathrm{~ms}$ ), the high-value area (red area) was in the left atrium of the posterior surface. For all subjects, the highcurrent areas of the early and late p-wave were in the right atrium of the anterior surface and the left atrium of the posterior surface, respectively.

\subsection{Maximal current value and direction of PCAM}

Table 1 shows the maximal current values and directions during early and late p-wave. Moreover, the ratio of maximal current value of early and late $\mathrm{p}$-wave appear in Table 1, which also shows the average maximal current values, directions, the averaged ratio of the maximal current value of the early $\mathrm{p}$-wave to the maximal current value for the fourteen subjects (male: twelve, female: two, average age \pm SD: $37 \pm 5$ ). During the early pwave stage, the maximal current values for all subjects was in a range from 17 to $84 \mathrm{pT} / \mathrm{m}$, and the average current value was $38 \pm 15 \mathrm{pT} / \mathrm{m}$. The maximal current direction during early p-wave was in a range of 17 to 95 degrees. The average current direction was $78 \pm 15$ degrees. During the late p-wave stage, the maximal current values for all subjects was in a range from 29 to

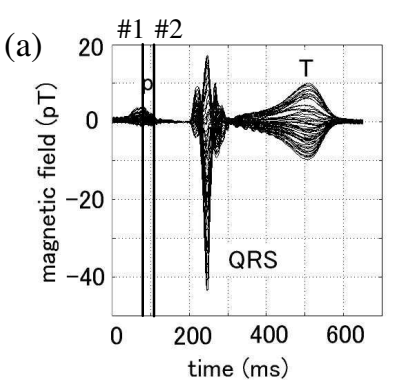

anterior

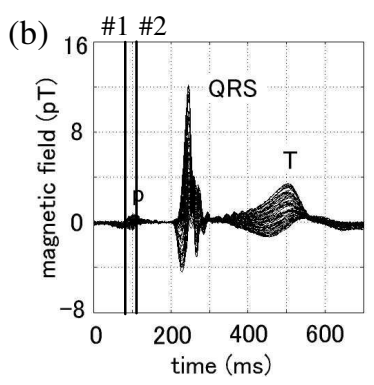

posterior
Figure 4. MCG waveforms for a healthy subject ((a) front plane, (b) back plane).

(a)

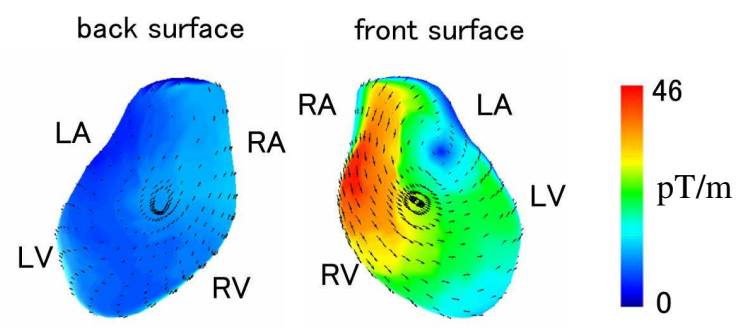

(b)

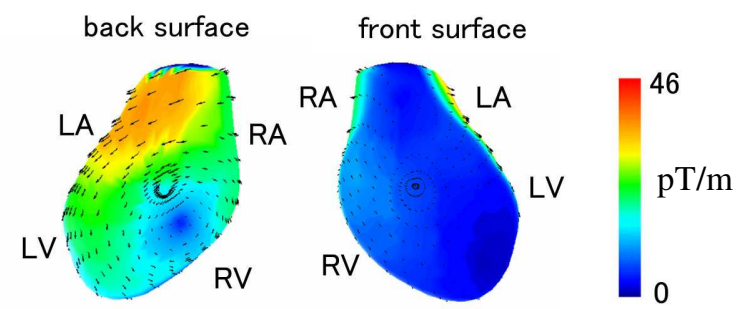

Figure 5. PCAMs for a healthy subject. (a) early p-wave (right atrial excitation), (b) late pwave (left atrial excitaton). RA: Right Atrium, LA: Left Atrium, RV: Right Ventricle, LV: Left Ventricle.

Table 1 Maximal current values and directions of early and late p-wave for fourteen healthy subjects.

\begin{tabular}{|c|c|c|c|c|c|}
\hline \multirow{2}{*}{ No. } & \multicolumn{2}{|c|}{ Maximal current value $(\mathrm{nT} / \mathrm{m}$} & \multirow{2}{*}{ ratio } & \multicolumn{2}{|c|}{ Maximal current direction (dearee) } \\
\hline & early $p$-wave & late p-wave & & early n-wave. & late p-wave \\
\hline N1 & 50 & 45 & 11 & 82 & 144 \\
\hline N2 & 39 & 68 & 0.6 & 96 & 151 \\
\hline N3 & 71 & 60 & 12 & 89 & 180 \\
\hline $\mathrm{N} 4$ & 17 & 26 & 07 & 82 & 161 \\
\hline N5 & 38 & 37 & 10 & 92 & 162 \\
\hline NG & 21 & 30 & 07 & 6.5 & 129 \\
\hline N7 & 22 & 40 & 0.6 & 79 & 171 \\
\hline N8 & 29 & 47 & 0.6 & 57 & 146 \\
\hline No & 23 & 31 & 07 & 24 & 185 \\
\hline N10 & 48 & 40 & 12 & 92 & 159 \\
\hline N11 & 84 & 61 & 14 & 90 & 84 \\
\hline $\mathrm{N} 12$ & 26 & 29 & 0.9 & 66 & 233 \\
\hline $\mathrm{N} 13$ & 29 & 43 & 07 & 17 & 165 \\
\hline N14 & 32 & 30 & 11 & 96. & 156 \\
\hline average & 378 & 41.9 & 0.9 & 777 & 164.8 \\
\hline $\mathrm{SD}$ & 147 & 104 & 0.2 & 152 & 16.9 \\
\hline
\end{tabular}


$68 \mathrm{pT} / \mathrm{m}$, and the current direction during early p-wave was in the range of the average current value of $42 \pm 10$ $\mathrm{pT} / \mathrm{m}$. The maximal current direction during the late $\mathrm{p}$ wave stage was in the range of 84 to 233 degrees. The average current direction was $165 \pm 17$ degrees. Furthermore, the averaged ratio of the maximal current value of right atrium to the maximal current value of the left atrium was $0.9 \pm 0.2$.

\section{Discussion}

To visualize the cardiac electrical current in an atrial myocardium, we projected $C A M \mathrm{~s}$, calculated from $M C G$ signals that corresponded to the p-wave, onto a 3-D standard heart model. The electrical activity in the p-wave should reflect right- and left atrial excitation [9]. The PCAMs during the p-wave are seen in Figs. 5 (a) and (b). During the early p-wave stage, the high-current area was in the right atrium of the model's anterior surface. In the later stage, the region of greatest activity shifted towards the left atrium of the model's posterior surface. Similar results during the p-wave for healthy subjects were reported using the anterior and posterior 2-D CAMs [10].

To evaluate the validity of the $P C A M$, we extracted the maximal current values and directions of early and late $\mathrm{p}$-wave for fourteen healthy subjects (Table 1). The averaged ratio of the maximal current value of early $p$ wave to the maximal current value of late p-wave was 0.9 \pm 0.2 . This indicates that the current value of right atrial excitation was almost equal to the current value of left atrial excitation. On the other hand, the averaged directions of maximal current of the early and late $p$-wave were quite different. Therefore, the anterior and posterior surfaces of the PCAM reflected the different excitation activity in the heart. These results show that the PCAM can produce a clear view of the actual electrical excitation in the left and right atrium during p-wave excitation.

\section{Conclusions}

We developed a PCAM method for use with a $3-D$ standard heart model. This method was applied to visualize early and late atrial excitation in fourteen healthy subjects. During early and late atrial excitation, high-current areas of all subjects were in the right atrium of the anterior surface and left atrium of the posterior surface, respectively. The averaged ratio of the maximal current value of the early $\mathrm{p}$-wave to the maximal current value of the late $p$-wave for all subjects was $0.9 \pm 0.2$. Furthermore, the averaged maximal current directions of the early and late p-wave were $78 \pm 15$ and $165 \pm 17$ degrees, respectively. We found that the PCAMs for healthy subjects reflected electrical excitation in the left and right atrium.

\section{References}

[1] Tsukada K, Haruta Y, Adachi A, Ogata H, Komuro T, Ito T, Takada Y, Kandori A, Noda Y, Terada Y, and Mitsui T: Multichannel SQUID system detecting tangential components of the cardiac magnetic field. Rev Sci Instrum 66: 5085, 1995

[2] Horigome H, Siono J, Shigemitsu S, Asaka M, Matsui A, Kandori A, Miyashita T, and TsukadaK: Detection of cardiac hypertrophy in the fetus by approximation of the current dipole using magnetocardiography. Pediatr Res 50: 242, 2001

[3] Miyashita T, Kandori A, and Tsukada K: Construction of tangential vectors from normal cardiac magnetic field components: Proc 20th Int Conf IEEE/EMBS (Hong Kong): 520, 1998

[4] Kandori A, Shimizu W, Yokokawa M, Kamakura S, Miyatake K, Murakami M, Miyashita T,Ogata K, and Tsukada K: Reconstruction of action potential of repolarization in patients with congenital long-QT syndrome. Phys Med Biol 49: 2103, 2004

[5] Kanzaki H, Nakatani S, Kandori A, Tsukada K, and Miyatake K: A new screening method to diagnose coronary artery disease using multichannel magnetocardiogram and simple exercise Basic Res Cardiol 98: 124, 2003

[6] Tsukada K, Mitsui T, Terada Y, Horigome H, and Yamaguchi I: Noninvasive visualization of multiple simultaneously activated regions on torso magnetocardiographic maps during ventricular depolarization. J Electrocardiol 32: 305, 1999

[7] Ogata K, Kandori A, Miyashita T, Tsukada K, Yamada S, Shimizu W, Nakatani S, Miyatake K, and Yamaguchi I: Projecting cardiac current images onto a 3-D standard heartmodel. Proc 20th Int Conf IEEE/EMBS (Cancun): 517,2003

[8] Nomura M, Nakaya Y, Saito K, Kishi F, Watatsuki T, Myoshi H, Nishikado B, Bando S, Ito S, Nishitani H, Wada M, Fujita S, and Tamura I: Noninvasive localization of accessory pathways by magnetocardiographic imaging. Clin Cardiol 17: 239, 1994

[9] Durrer D, van Dam R, Freud GE, Janse MJ, Meijler FL, and Arzbaecher RC: Total Excitation of the isolated human heart. Circulation 41: 899, 1970

[10] Sato M, Terada Y, Mitsui T, Miyashita T, Kandori A and Tsukada K: Visualization of atrialexcitation by magnetocardiogram. Int J Card Imag 18: 305, 2002

Address for correspondence

Kuniomi Ogata

1-280, Higashi-koigakubo, Kokubunji city, Tokyo, 185-8601, Japan

k-ogata@rd.hitachi.co.jp 